\title{
Study of the Interaction of Citrate-capped Hollow Gold Nanospheres with Metal Ions
}

\author{
Miller $\mathrm{A}^{1}$, Adams $\mathrm{S}^{2}$, Zhang $\mathrm{JZ}^{2 *}$ and Wang $\mathrm{L}^{1 *}$ \\ ${ }^{1}$ Department of Chemistry and Biochemistry, Kettering University, Flint, MI 48504, USA \\ ${ }^{2}$ Department of Chemistry and Biochemistry, University of California, Santa Cruz, CA 95064, USA
}

\begin{abstract}
Understanding the interaction between metal nanostructures and common metal ions is essential for biomedical applications of metal nanostructures including imaging and therapy. A systemic study of the interaction of citratecapped hollow gold nanospheres (HGNs), a unique metal nanostructure that has demonstrated high efficiency for photothermal ablation (PTA) therapy of cancer cells, with common metal ions: $\mathrm{Na}^{+}, \mathrm{Ca}^{2+}, \mathrm{Mg}^{2+}, \mathrm{Cu}^{2+}, \mathrm{Zn}^{2+}$, and $\mathrm{Al}^{3+}$ was carried out with the goal to determine how these metal ions may affect the properties and thereby performance of HGNs in biomedical applications. Specifically, the study focuses on the issue of metal ion induced aggregation of HGNs since aggregation can strongly influence the optical and photothermal properties of the HGNs. The level of HGNs aggregation caused by interaction with metal ions was found to depend on the nature and concentration of the metal ions present in the solution as well as the properties of the HGNs. The larger the stability constant of the metal ion citrate complex $\left(\log \mathrm{K}_{1}\right)$ is, the stronger the interaction between the metal ions and the HGNs was found to be. Lower HGN concentrations and higher levels of dispersion were correlated to enhanced sensitivity of the HGNs towards metal ion induced aggregation. The results demonstrate that metal ions can strongly affect how nanostructures like HGNs can be used in biomedical applications including imaging and therapy.
\end{abstract}

Keywords: Nanospheres; Photothermal; Nanostructures

\section{Introduction}

Gold nanostructures have attracted a great deal of interest due to their unique optical properties including localized surface plasmon resonance (LSPR), which make them useful for a variety of biomedical applications including drug delivery, sensing, imaging and photothermal therapies [1-9]. The extent of biomedical applicability of these nanostructures depends strongly upon their stability in biological solutions [10-14]. Parameters such as size, shape, surface properties, and the dielectric constant of the surrounding medium influence the stability of gold nanostructures [10-14]. In particular, surface bound organic species play an important role in the generation or prevention of aggregation of nanostructures [15-19].

Since metal ions are common components of biological solutions, it is important to determine the factors affecting the interaction between various metal ions and gold nanostructures that can lead to differences in stability and aggregation. While aggregation resulting from the formation of coordination complexes between metal ions and ligands on the surface of gold nanostructures may be undesirable for certain applications like photo thermal therapies, metal ion induced aggregation may be useful in other applications. For example, the LSPR shift resulting from metal ion induced aggregation has been exploited for use in the colorimetric detection of metal ions [20-22].

A special class of nanostructure, hollow gold nanospheres (HGNs), has been developed [23-28]. HGNs have the unique combination of spherical shape, small size $(<100 \mathrm{~nm})$, biological compatibility, and chemical inertness, making them excellent candidates for biological applications [29]. By controlling the aspect ratio between the hollow core and shell diameter HGNs can be tuned through synthesis to absorb any wavelength within visible region and out to the near infrared (NIR) of the EM spectrum. NIR absorbing nanostructures are of particular importance since blood and tissue are easily penetrated by light in this region. HGNs have been successfully used in sensing applications employing surface enhanced Raman scattering (SERS) $[17,30-32]$ and photothermal ablation therapy applications [26,33].
HGNs have also been utilized in drug delivery [34,35], including NIR light triggered release of doxorubicin for photothermal chemotherapy [36] and in-vivo biological imaging [7,37]. However, the possible effect of metal ions on the properties and stability of HGNs have not yet been explored or well understood.

Citrate is a common ligand used in both the synthesis of gold nanostructures and as a capping agent to prevent aggregation $[24,38,39]$. However, in the presence of metal ions, gold nanostructures capped with citrate [40] or other chelating ligands such as amino acids [20-22] tend to aggregate due to the coordination complexes formed between the capping agent and metal ions. In fact, this metal ion induced nanoparticle aggregation has been explored to develop inexpensive metal ion detection methods using citrate and amino acid capped gold nanoparticles [20-22,40]. In this study, citrate was employed as a stabilizing agent because of its enhanced negative charge when compared to amino acids, e.g., cysteine at a neutral $\mathrm{pH}$ $[41,42]$. The structures and $\mathrm{pK}_{\mathrm{a}}$ values of citrate and cysteine are shown in Figure 1. According to the $\mathrm{pK}_{\mathrm{a}}$ values, citrate has a negative three charge at neutral $\mathrm{pH}$ because all three carboxyl groups are deprotonated [41], which prevents the particles from aggregating due to electronic repulsion. In comparison, at neutral $\mathrm{pH}$, cysteine has a positively charged $\mathrm{NH}_{3}{ }^{+}$group and a negatively charged - $\mathrm{COO}^{-}$group [42]. This means that even after the thiol group of cysteine is deprotonated due

*Corresponding authors: Jin Z Zhang, Department of Chemistry and Biochemistry University of California, Santa Cruz, CA 95064, USA, Tel: 1 310-825-4219; E-mail: zhang@ucsc.edu

Lihua Wang, Department of Chemistry and Biochemistry, Kettering University, Flint, MI 48504, USA, Tel: 1 310-825-4219; E-mail: Iwang@kettering.edu

Received April 12, 2016; Accepted April 25, 2016; Published April 30, 2016

Citation: Miller A, Adams S, Zhang JZ, Wang L (2016) Investigation into Drug Solubilisation Potential of Sulfonated Calix(4)Resorcinarenes. J Nanomed Nanotechnol 7: 371. doi:10.4172/2157-7439.1000371

Copyright: ( 2016 Miller A, et al. This is an open-access article distributed under the terms of the Creative Commons Attribution License, which permits unrestricted use, distribution, and reproduction in any medium, provided the original author and source are credited. 
to its binding to gold, cysteine only has an overall charge of negative one, which can lead to aggregation of the cysteine capped particles even without the presence of metal ions [43]. Additionally, cysteine is known to generate disulfide bonds which may promote aggregation. A study on the interaction of citrate capped gold nanoparticles and different salt solutions have been reported [44]. Color change was used to determine the end points of the titrations of nanoparticles with various salt solutions [44]. However, since metal ion induced color change of gold nanoparticles is a gradual process, it is difficult to pinpoint the exact end point of the titration.

In this study, we have conducted a systematic study of interaction between the HGNs and metal ions. The strength of the interaction between the HGNs and metal ions was determined by the slopes of calibration curves that relate the extent of aggregation of HGNs with the concentrations of metal ions in solution. The extent of aggregation of HGNs was determined using the ratio of absorbance at the $\lambda$ of the non-aggregated HGNs solution to the absorbance at the $\lambda_{\max }$ of the aggregated HGNs solution. We found that the extent of metal ioninduced aggregation depends on the nature and the concentration of the metal ion, as well as the concentration and the level of dispersion of the HGNs. The results of this study provide information that may be used in the design of HGNs that are stable in electrolyte solutions and the development of HGNs based colorimetric methods of metal ion analysis.

\section{Experimental}

\section{Materials and equipment}

The following chemicals were used in the synthesis of HGNs without further purification: $\mathrm{CoCl}_{2}$ (Certified ACS Reagent, Fisher Scientific), Sodium Citrate Dihydrate (Analytical Reagent, Mallinckrodt), $\mathrm{HAuCl}_{4}$ (99.999\%, Aldrich), $\mathrm{NaBH}_{4}$ (granular, 99.99\%, Sigma). The glassware used for the synthesis was cleaned by Aqua Regia (three parts of concentrated $\mathrm{HCl}$ to 1 part of concentrated $\mathrm{HNO}_{3}(\mathrm{v} / \mathrm{v})$ ) and then rinsed thoroughly with deionized water and ultrapure water of at least $15 \mathrm{M} \Omega$. The reactions were carried out in ultrapure water that was degassed with nitrogen.

The following chemicals were used without further purification to prepare the metal ion solutions for the study of the interaction of HGNs with metal ions: $\mathrm{NaNO}_{3}$ (Certified ACS Reagent, Fisher Scientific), $\mathrm{Ca}\left(\mathrm{NO}_{3}\right)_{2} \cdot 4 \mathrm{H}_{2} \mathrm{O}$ (ACS Reagent, GFS Chemicals), $\mathrm{Cu}\left(\mathrm{NO}_{3}\right)_{2} \cdot 3 \mathrm{H}_{2} \mathrm{O}$ (ACS Reagent, ACROS Organics), $\mathrm{ZnSO}_{4} \cdot 7 \mathrm{H}_{2} \mathrm{O}$ (Certified ACS Reagent, Fisher Scientific), $\mathrm{CuSO}_{4} 5 \mathrm{H}_{2} \mathrm{O}$ (Certified ACS Reagent, Fisher Scientific), $\mathrm{Al}\left(\mathrm{NO}_{3}\right)_{3} \cdot 9 \mathrm{H}_{2} \mathrm{O}$ (ACS Reagent, GFS Chemicals), $\mathrm{MgSO}_{4}$ (Analytical Reagent, Mallinckrodt, Inc.).

UV-Vis spectra were taken using Cary 50 Bio UV-Vis Spectrophotometer by Varian. Scanning Electron microscopy images were obtained using an FEI Quanta 3-D dual beam microscope with an accelerating voltage of $5.00 \mathrm{kV}$.

\section{Synthesis of citrate-capped hollow gold nanospheres}

The synthesis of HGNs was carried out according to a previously published method with minor modifications [24]. Cobalt nanoparticle templates were generated by reducing $\mathrm{CoCl}_{2}$ with sodium borohydride in an oxygen free environment. Then a galvanic replacement reaction was employed to oxidize the sacrificial template by the $\mathrm{Au}^{3+}$ ions in the $\mathrm{HAuCl}_{4}$ salt, which has a higher reduction potential than cobalt(II) ions. The reaction takes place according to the following equation:

$3 \mathrm{Co}(0)+2 \mathrm{Au}(\mathrm{III}) \rightarrow 3 \mathrm{Co}(\mathrm{II})+2 \mathrm{Au}(0)$
Where it can be seen that for every three Co atom oxidized, two gold atoms are formed. Since the generation of gold shell deposition is slower than the oxidation of cobalt, the result of this process is the formation of a hollow structure with a polycrystalline gold shell.

The detailed synthesis procedure is as follows: $200 \mu \mathrm{L}$ of $0.10 \mathrm{M}$ sodium citrate and $50 \mu \mathrm{L}$ of $0.40 \mathrm{M} \mathrm{CoCl}_{2}$ solutions were added to $50 \mathrm{~mL}$ of degased ultrapure water. The solution was degased with nitrogen gas for about $45 \mathrm{~min}$. $500 \mu \mathrm{L}$ of freshly prepared degased 0.1 $\mathrm{M} \mathrm{NaBH}_{4}$ was added to the solution and $200 \mu \mathrm{L}$ of additional sodium citrate solution was injected into the solution as soon as the solution started to turn brown. The solution was degased for an additional 45 $\min$. Then, $30 \mathrm{~mL}$ of the solution was transferred to a degased $10 \mathrm{~mL}$ $0.15 \mathrm{mM} \mathrm{HAuCl}_{4}$ solution under vigorous stirring. The solution was then opened to the atmosphere and stirred continuously until the color changed from brown to red or purple depending on the wavelength of absorption.

The resulting HGNs were isolated and washed with $0.6 \mathrm{mM}$ sodium citrate by centrifugation and reconstituted twice. Finally, the HGNs were re-suspended in fresh citrate and stored. Immediately prior to use in the binding study, the HGNs were isolated by centrifugation and washed twice with ultrapure water to remove any remaining unbound citrate.

\section{Study of the Interaction of citrate-capped HGNs with metal ions}

The interaction of citrate-capped HGNs with a variety of metal ions, including $\mathrm{Na}^{+}, \mathrm{Ca}^{2+}, \mathrm{Zn}^{2+}, \mathrm{Mg}^{2+}, \mathrm{Cu}^{2+}$ and $\mathrm{Al}^{3+}$, was studied using UV/Vis absorption spectroscopy. Both metal nitrates and metal sulfates were used in this study and no significant difference was found in the extent of aggregation of HGNs generated due to the presence of either anion. In a typical experiment, $900 \mu \mathrm{L}$ of the HGNs solution was placed in a cuvette. A spectrum was then collected across the visible region from $350-800 \mathrm{~nm}$. The HGNs were then titrated with small increments of a $10 \mathrm{mM}$ metal ion solution. A new spectrum was taken every 5 minutes after each addition of the metal ion solution until the SPR stabilized and no longer shifted. Usually, 5-20 min was needed to allow the reaction to reach equilibrium. The ratio of absorbance at the $\lambda_{\max }$ of the non-aggregated HGNs solution to the absorbance at $750 \mathrm{~nm}$ or $700 \mathrm{~nm}\left(\lambda_{\text {max }}\right.$ of the aggregated HGNs solution) after each addition of metal ion was determined and graphed against the concentration of the metal ion. The absorbance ratio was used instead of absorbance at one wavelength in order to eliminate the effect of the HGNs concentration on the slope of the calibration curve. The slopes of the calibration curves were used to compare the strength of the interaction between the HGNs and various metal ions.

\section{Results and Discussions}

\section{Characterization of citrate-capped hollow gold nanospheres}

The citrate-capped HGNs used in the study were characterized by UV/Vis spectroscopy and scanning electron microscope. The absorption maximum of the HGN SPR depends mainly on the aspect ratio between the solvent filled hollow core diameter and the diameter of the gold shell. The larger the diameter of the core is and the thinner the gold shell is, the more red shifted the SPR will be [24]. The absorption maxima of the HGNs used in this study ranged from $550 \mathrm{~nm}$ to 590 $\mathrm{nm}$. Typical UV/Vis spectral changes induced by the introduction of the metal ions, is shown in Figure 2 where $\mathrm{Cu}^{2+}$ was used as an example.

It can be seen that when the HGNs are suspended in solution, without the addition of any metal ions, the absorption peak is centered 
Citation: Miller A, Adams S, Zhang JZ, Wang L (2016) Investigation into Drug Solubilisation Potential of Sulfonated Calix(4)Resorcinarenes. J Nanomed Nanotechnol 7: 371. doi:10.4172/2157-7439.1000371

Page 3 of 7<smiles>O=C([O-])C(O)(C[O-])C[O-]</smiles>

Citrate pKa values ${ }^{40}: 3.13,4.76,6.40$

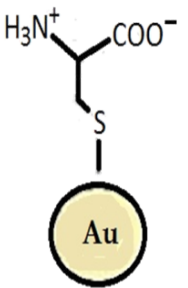

Cysteine pKa values $^{41}: 1.70$, , 8.3, 10.8

Figure 1: Structures and $\mathrm{pK}_{\mathrm{a}}$ values of citrate and cysteine.

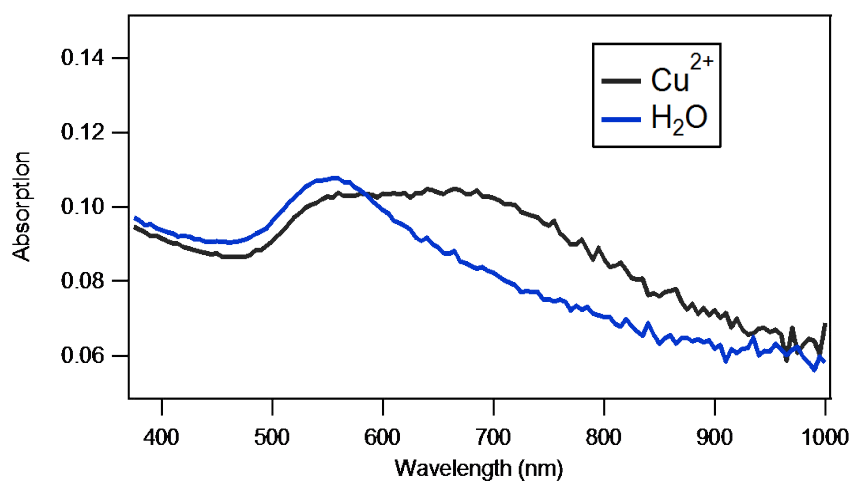

Figure 2: Typical UV-Vis spectra of HGNs before and after introducing a metal ion. The narrow, symmetrical peak corresponds to the HGN sample before the addition of $\mathrm{Cu}^{2+}$ ions. After exposure to $\mathrm{Cu}^{2+}$ ions, a new broad band peaked around $700 \mathrm{~nm}$ appeared, which is indicative of aggregation of HGNs.

at $\sim 550 \mathrm{~nm}$. This peak is symmetric in shape and has a narrow full width half maximum (FWHM), which is indicative of a homogeneously sized and monodispersed nanoparticle population in solution. Once the metal ions are added the spectrum both red shifts and broadens. In addition to a shoulder near the original absorption of $\sim 550 \mathrm{~nm}$, there is a new absorption peak at $\sim 650 \mathrm{~nm}$. This indicates polydispersity of size among the HGNs in solution. Additionally, the absorption FWHM extends several hundred nanometers indicative of aggregation.

Figure 3 shows representative SEM images of HGNs before and after exposure to $\mathrm{Cu}^{2+}$ metal ions. Before the HGNs were exposed to metal ions, the average particle diameter, including core and shell, was determined to be $17.7 \pm 2.0 \mathrm{~nm}$ (Figure $3 \mathrm{a}$ ). The particles were uniform in size and shape and monodispersed within the solution. Figure $3 \mathrm{~b}$ is representative of the induced aggregation generated by the exposure of the HGNs to $\mathrm{Cu}^{2+}$ ions. Once in contact with the metal ions, the HGNs became aggregated into a variety of large, irregularly shaped clusters.

\section{Interaction of citrate-capped HGNs with common metal ions}

Typical changes in the absorption spectrum of the citrate-capped HGNs with increasing concentration of metal ions are shown in Figure 4 using $\mathrm{Cu}^{2+}$ and $\mathrm{Ca}^{2+}$ as examples. As the metal ion concentration increases, the absorption maximum is shifted to a longer wavelength, which is consistent with the aggregation of the HGNs. Concentration dependence of the absorbance ratio, absorbance at $\lambda_{\max }$ of nonaggregated HGNs to the absorbance at the $\lambda_{\max }$ of aggregated HGNs, was used to determine the extent of metal ion binding with the HGNs.

Effects of the properties of the HGNs on the calibration curve: It was observed that the calibration curves were dependent on the concentration and the level of dispersion of the HGNs before the addition of metal ions. The concentration of HGNs did not affect the slope of the calibration curve, but did affect the linear range of the calibration curve (Table 1). The calibration curves are based on absorbance ratio instead of absorbance at a particular wavelength, which eliminates the dependence of the slope of the calibration curve on the concentration of the HGNs. However, the detection limit and the linear concentration range of the calibration curve did vary with the concentration of the HGNs used. It was found that the higher the concentration of the HGNs is, the higher both the lower and the upper limits of the linear concentration range are (Table 1). The limits of the linear range increase because a higher concentration of HGNs requires a higher concentration of metal ions to reach the same level of aggregation.

The slope of the calibration curve was independent of the concentration of the HGNs and was correlated to the level of HGN dispersion before the introduction of metal ions. The absorbance ratio of the HGNs in the absence of metal ions is a good indicator of the level of dispersion of the HGNs, with a higher absorbance ratio indicating greater dispersity. Table 2 shows the results of $\mathrm{Ca}^{2+}$ and $\mathrm{Mg}^{2+}$ binding studies using two different batches of HGNs having different levels of dispersion but the same concentration. It can be seen that the more dispersed HGNs generated larger calibration curve slopes. This shows that the more dispersed the HGNs are, the more sensitive they are to the binding of metal ions.

Effects of the nature of the metal ions: The calibration curves were determined for the binding of HGNs with a variety of common metal ions including $\mathrm{Na}^{+}, \mathrm{Ca}^{2+}, \mathrm{Zn}^{2+}, \mathrm{Cu}^{2+}, \mathrm{Mg}^{2+}$ and $\mathrm{Al}^{3+}$. It was observed that the slope of the calibration curve was dependent on the nature of the metal ions when HGNs of the similar size, level of dispersion and concentration were used. The slope of the calibration curve indicates the sensitivity of HGNs aggregation to the change in metal ion concentration, which in turn indicates the binding strength between the HGNs and the metal ions.

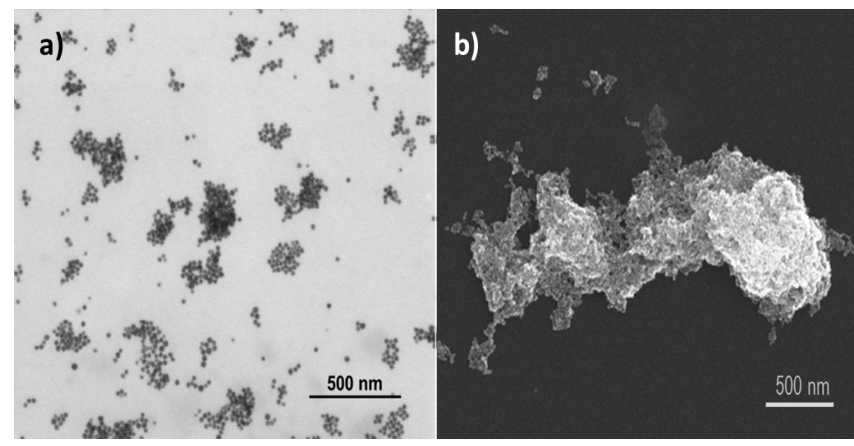

Figure 3: SEM image of HGN's before and after exposure to $\mathrm{Cu}^{2+}$ metal ions. Image (a) shows HGNs suspended in water. The nanoparticles are monodispersed with an average diameter, including both core and shell, of $17.6 \pm 2.0 \mathrm{~nm}$. Image $\mathrm{b}$ shows the aggregation of HGNs following titration with $\mathrm{Cu}^{2+}$ ions. 


\begin{tabular}{|c|c|c|c|c|}
\hline Metal lons & \multicolumn{2}{|c|}{$\mathbf{C a}^{2+}$} & \multicolumn{2}{|c|}{$\mathbf{C u}^{2+}$} \\
\hline $\begin{array}{c}\lambda_{\max } \text { of nonaggregated } \\
\text { HGNSs }\end{array}$ & $575 \mathrm{~nm}$ & $578 \mathrm{~nm}$ & $575 \mathrm{~nm}$ & $587 \mathrm{~nm}$ \\
\hline Absorbance at $\lambda_{\max }$ & 0.0861 & 0.136 & 0.0800 & 0.1818 \\
\hline $\begin{array}{c}\text { Slope of the } \\
\text { Calibration Curve }\end{array}$ & -0.0046 & -0.0048 & -0.015 & -0.014 \\
\hline $\begin{array}{c}\text { Absorbance Ratio } \\
\text { without the presence } \\
\text { of metal ion }\end{array}$ & 3.31 & 3.85 & 3.90 & 3.97 \\
\hline $\begin{array}{c}\text { Linear Concentration } \\
\text { Range of the } \\
\text { Calibration Curve }\end{array}$ & $110-530$ & $200-700$ & $0-100$ & $0-200$ \\
\hline
\end{tabular}

Table 1: Effects of HGNs concentration on the linear concentration range of the calibration curve.

\begin{tabular}{|c|c|c|c|c|}
\hline Metal lons & \multicolumn{2}{|c|}{$\mathrm{Ca}^{2+}$} & \multicolumn{2}{|c|}{$\mathrm{Mg}^{2+}$} \\
\hline $\begin{array}{c}\lambda_{\max } \text { of nonaggregated } \\
\text { HGNSs }\end{array}$ & $560 \mathrm{~nm}$ & $561 \mathrm{~nm}$ & $561 \mathrm{~nm}$ & $560 \mathrm{~nm}$ \\
\hline Absorbance at $\lambda_{\max }$ & 0.103 & 0.106 & 0.103 & 0.118 \\
\hline $\begin{array}{c}\text { Slope of the } \\
\text { Calibration Curve }\end{array}$ & -0.0158 & -0.0224 & -0.007 & -0.0086 \\
\hline $\begin{array}{l}\text { Y-Intercept of the } \\
\text { Calibration Curve }\end{array}$ & 5.85 & 7.59 & 5.64 & 7.98 \\
\hline
\end{tabular}

Table 2: Effects of the level of dispersion of the HGNs on the calibration curve.

Interaction of citrate-capped $\mathrm{HGNs}$ with $\mathrm{Na}^{+}$was studied in the concentration range of $0-20 \mathrm{mM}$ and the resulting calibration curve are shown in Figure 5. The calibration curve is linear up to about $8.0 \mathrm{mM}$ $\mathrm{Na}^{+}$and the slope of the line is $-0.0008 \mu \mathrm{M}^{-1}$. Calibration curves for $\mathrm{Ca}^{2+}$ and $\mathrm{Cu}^{2+}$ are shown in Figure 6. The slope of the linear portion of the graph for $\mathrm{Cu}^{2+}\left(-0.0623 \mu \mathrm{M}^{-1}\right)$ is greater than that of $\mathrm{Ca}^{2+}(-0.0224 \mu \mathrm{M}$ $\left.{ }^{1}\right)$. Both are much higher than that of $\mathrm{Na}^{+}$due to the charge difference of the ions. The slopes of the calibration curves for $\mathrm{Mg}^{2+}(-0.0084$ $\left.\mu \mathrm{M}^{-1}\right)$ and $\mathrm{Zn}^{2+}\left(-0.0168 \mu \mathrm{M}^{-1}\right)$ have also been determined (Table 1). According to the results, the order of increasing strength of interaction of the HGNs with the divalent metal ions is $\mathrm{Mg}^{2+}<\mathrm{Zn}^{2+}<\mathrm{Ca}^{2+}<\mathrm{Cu}^{2+}$, which in general correlates well with the order of increasing binding constant of metal citrates except for $\mathrm{Zn}^{2+}: \mathrm{Mg}^{2+}\left(\log \mathrm{K}_{1}=2.8\right)<\mathrm{Ca}^{2+}$ $\left(\log \mathrm{K}_{1}=3.5\right)<\mathrm{Zn}^{2+}\left(\log \mathrm{K}_{1}=4.5\right)<\mathrm{Cu}^{2+}\left(\log \mathrm{K}_{1}=6.1\right)$ [44]. It is not clear why $\mathrm{Zn}^{2+}$ does not follow the trend.

Plasmon resonance shift was observed for $\mathrm{Al}^{3+}$ in the concentration range of $0-16 \mu \mathrm{M}$, which is about ten times lower than that observed for $\mathrm{Cu}^{2+}$ and other divalent ions. The linear range of the calibration curve is $0-11 \mu \mathrm{M} \mathrm{Al}^{3+}$ (Figure 7). The slope of the line $\left(-0.501 \mu \mathrm{M}^{-1}\right)$ is much greater than that of the divalent ions studied due to the higher charge of $\mathrm{Al}^{3+}$ (Table 3).

Effects of the nature of the anion on the interaction of citratecapped HGNs with $\mathrm{Cu}^{2+}$ : The interaction of citrate-capped HGNs with $\mathrm{Cu}\left(\mathrm{NO}_{3}\right)_{2}$ was compared to that of $\mathrm{CuSO}_{4}$ to determine the effects of different anions on the interaction of metal ions with the HGNs. The absorbance ratio vs. concentration graphs for $\mathrm{CuSO}_{4}$ and $\mathrm{Cu}\left(\mathrm{NO}_{3}\right)_{2}$ are shown in Figure 8. It can be seen that the slope and the linear range for both graphs are the same. Therefore, no significant difference was observed for the interaction of $\mathrm{HGNs}$ with $\mathrm{Cu}^{2+}$ in the presence of either $\mathrm{NO}_{3}{ }^{-}$or $\mathrm{SO}_{4}{ }^{2-}$ counter ion. This result is consistent with that of the previous study of the interaction of metal ions with citrate capped gold nanoparticles $[44,45]$.

\section{Feasibility of using HGNSs for metal ion detection}

Colorimetric analysis of low levels of metal ions based on metal ion induced aggregation of gold nanostructures has the advantages including inexpensive instrumentation and easier to perform compared to atomic absorption methods. And it has the ability to detect metal ions such as $\mathrm{Ca}^{2+}, \mathrm{Mg}^{2+}$, and $\mathrm{Al}^{3+}$ that are colorless without using complex ligands to generate fluorescence or other optical signals. Gold nanoparticles capped with amino acids and citrates have been used for the detection of various metal ions [20-22,40].

Unlike gold nanoparticles, HGNs can be synthesized to absorb light over a much wider range of wavelengths including the NIR region where cloudy solutions or biological tissues are more transparent [23,27-29,34]. This study also provides some new insight into the feasibility of using citrate-capped HGNs for metal ion detection. First, the sensitivity and detection limit of the HGNs used in this study for $\mathrm{Cu}^{2+}$ and $\mathrm{Al}^{3+}$ detection were determined to be comparable to that of gold nanoparticles reported in the literature $[20,40]$. In addition, the sensitivity and detection limits were found to vary with the concentration and the degree of dispersion of the HGNs used. When using HGNs of the same size and level of dispersion at similar concentration, the sensitivity of detection increases with the charges of the metal ion and the stability of the metal-citrate complex (Table 3 and Figure 9).

Correlating the strength of metal-ion-HGN interactions with the stability constants of metal-capping agents (Table 3 and Figure 9) provides a path toward predicting the relative strength of the interaction between HGNs and different metal ions. The relative strength of HGNmetal ion interactions can also be used to identify which metal ion is present in a particular sample. For example, the citrate-capped HGNs used in this study are about 623 times more sensitive to $\mathrm{Al}^{3+}$ than to $\mathrm{Na}^{+}$ (Table 3). Therefore, the presence of $\mathrm{Na}^{+}$at the same concentration as $\mathrm{Al}^{3+}$ will cause an approximate $0.1 \%$ error. However, the presence of a divalent ion, such as $\mathrm{Cu}^{2+}$, at the same concentration would cause an error about $12 \%$ to $13 \%$ because $\mathrm{Al}^{3+}$ is only eight times more sensitive than $\mathrm{Cu}^{2+}$. In addition, a sample with much higher concentration of a particular metal ion than other metal ions present may be analyzed by this method.

\section{Conclusions}

The results of this study indicate that the interaction between citrate-capped HGNs and metal ions depends on the nature and the concentration of the metal ions, as well as the concentration and the level of dispersion of HGNs before the addition of metal ions. In general, the strength of interaction between the HGNs and metal ions, represented by the slope of the calibration curve, correlates to the stability constant $\left(\log \mathrm{K}_{1}\right)$ of the metal-citrate complex. It was found that the larger the $\log \mathrm{K}_{1}$ is, the stronger the interaction is, and the larger the slope of the calibration curve is. This type of correlation could be used to predict and compare the relative strength of interaction between citratecapped HGNs and different metal ions and to determine the stability of the HGNs in a solution with a particular metal ion composition.

\begin{tabular}{|c|c|c|c|}
\hline Metal Ion & $\begin{array}{c}\text { Slope }(\boldsymbol{\Delta} \text { abs } \\
\boldsymbol{\mu M})\end{array}$ & LogK $_{\mathbf{1}}$ & Linear Range $(\boldsymbol{\mu M})$ \\
\hline Sodium(I) & -0.0008 & ---- & $0-8,000$ \\
\hline Magnesium(II) & -0.0086 & 2.8 & $0-620$ \\
\hline Calcium(II) & -0.0224 & 3.5 & $0-270$ \\
\hline Zinc(II) & -0.0169 & 4.5 & $0-380$ \\
\hline Copper(II) & -0.0623 & 6.1 & $0-90$ \\
\hline Aluminum(III) & -0.4991 & 11.7 & $0-11$ \\
\hline
\end{tabular}

"Citrate-Capped HGNs of similar size, level of dispersion, and concentration were used.

Table 3: Relationship between the Binding Strength of Citrated-Capped HGNs with Metal lons and the Stability Constants $\left(\operatorname{LogK}_{1}\right)$ of Metal-Citrate Complexes. 


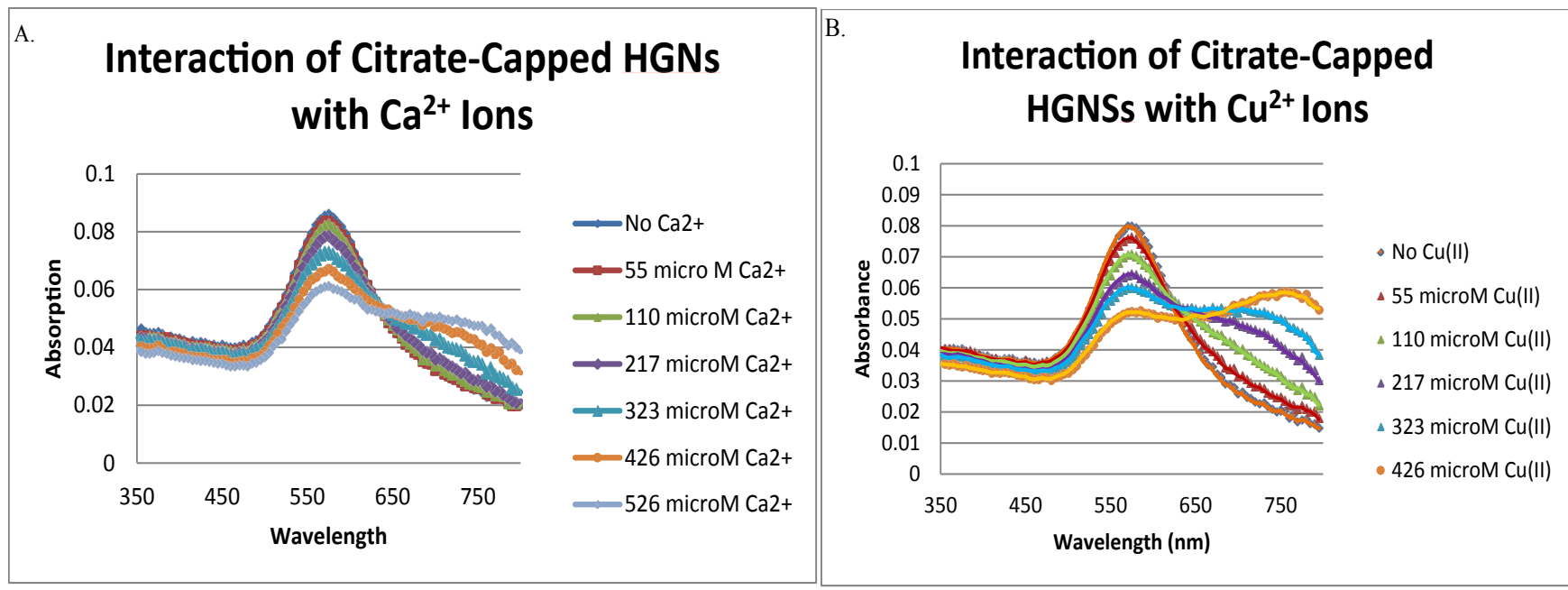

Figure 4: Plasmon resonance spectra of citrate-capped $\mathrm{HGNs}$ at the presence of various concentrations of $\mathrm{Ca}^{2+}$ ion $(\mathrm{A})$ and $\mathrm{Cu}^{2+}(\mathrm{B})$.
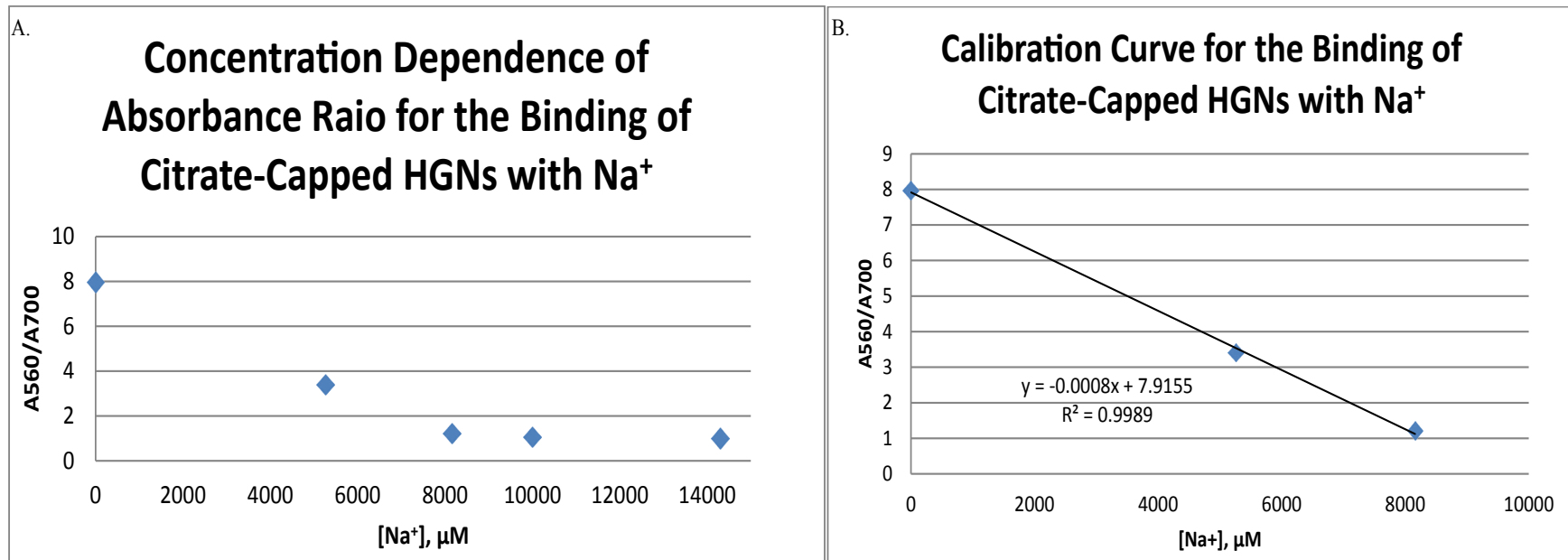

Figure 5: Concentration dependence of the absorbance ratio and linear calibration curve for the binding of citrate-capped $\mathrm{HGNs}$ with $\mathrm{Na}^{+}$.
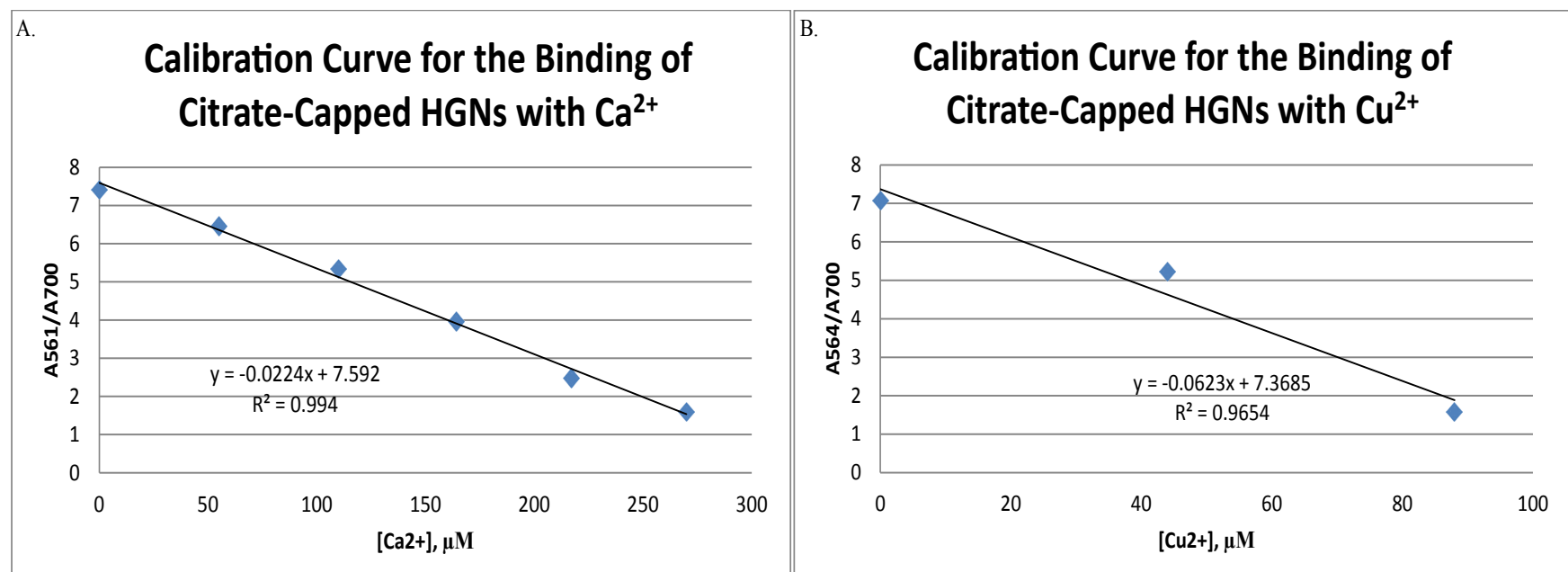

Figure 6: Calibration curves for the binding of citrate-capped $\mathrm{HGNSs}$ with $\mathrm{Ca}^{2+}(\mathrm{A})$ and $\mathrm{Cu}^{2+}(\mathrm{B})$. 
Citation: Miller A, Adams S, Zhang JZ, Wang L (2016) Investigation into Drug Solubilisation Potential of Sulfonated Calix(4)Resorcinarenes. J Nanomed Nanotechnol 7: 371. doi:10.4172/2157-7439.1000371

Page 6 of 7

In addition, lower HGN concentration and higher level of dispersion of HGNs increase the sensitivity of the HGNs to metal ion induced aggregation. The results have important implications in the use of HGNs or similar metal nanostructures for biomedical applications such as sensing, imaging, drug delivery, and cancer therapy. In addition, the results provide some new insights for the development of colorimetric analysis of metal ions using HGNs.

\section{Acknowledgement}

The authors would like to thank the undergraduate research fund of the Department of Chemistry and Biochemistry of Kettering University (The Cooper Tire Research Fund) for the financial support, and the W. M. Keck Center of Nanoscale Optofluidics at UCSC for using the FESEM. JZZ is grateful to Delta Dental Health Associates and Smart Technologies for financial support.

\section{Calibration Curve for the Binding of Citrate-Capped HGNs with $\mathrm{Al}^{3+}$}

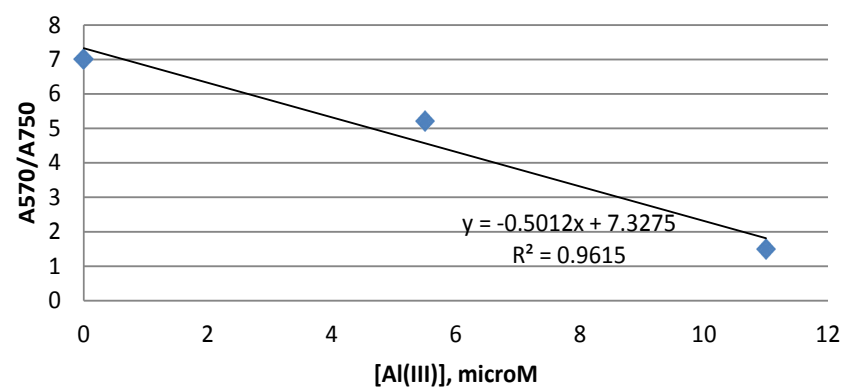

Figure 7: Concentration dependence and linear calibration curve of the interaction of $\mathrm{Al}^{3+}$ with citrate-capped HGNs.

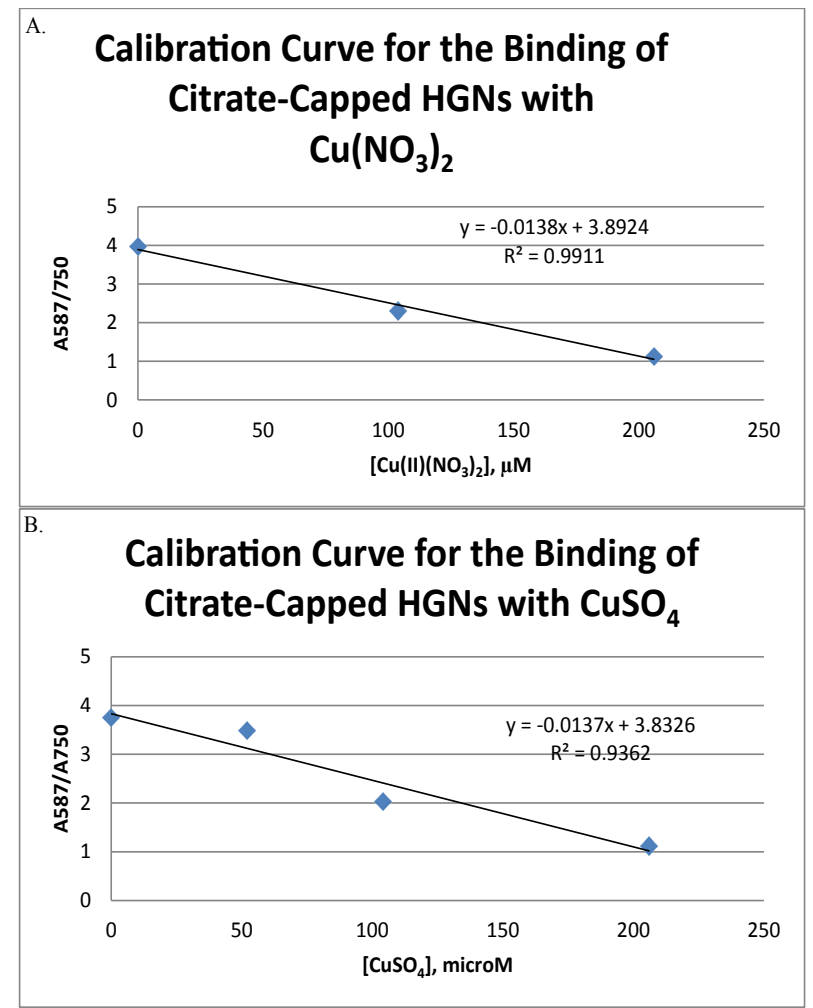

Figure 8: Calibration curve of the interaction of $\mathrm{Cu}^{2+}$ with citrate-capped HGNSs using $\mathrm{CuSO}_{4}(\mathrm{~A})$ and $\mathrm{Cu}\left(\mathrm{NO}_{3}\right)_{2}$.
Relationship between the Binding Strength of the CitrateCapped HGNs with Metal lons and the Stability Constants of Metal Citrate Complexes

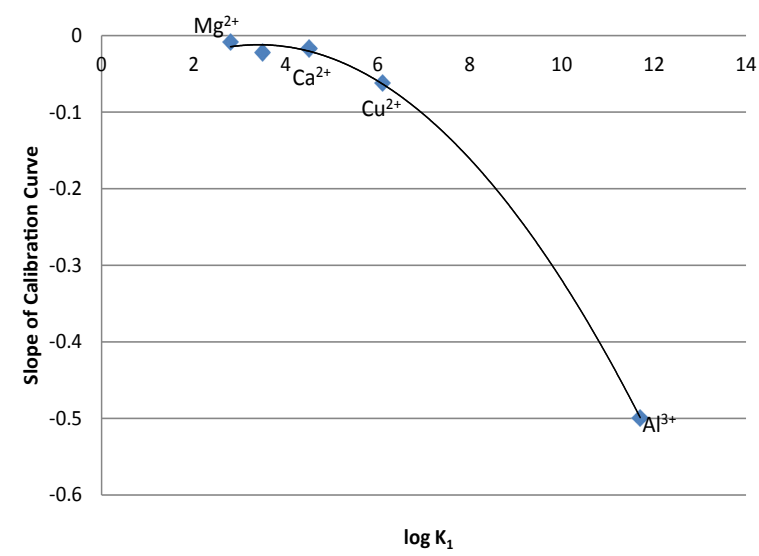

Figure 9: The dependence of binding strength between the HGNs and metal ions on the stability constants $\left(\operatorname{LogK}_{1}\right)$ of metal citrate complexes.

\section{References}

1. Jain PK, Huang X, El-Sayed IH, El-Sayed MA (2008) Noble Metals on the Nanoscale: Optical and Photothermal Properties and Some Applications in Imaging, Sensing, Biology, and Medicine. Accounts of Chemical Research 41 : 1578-1586.

2. Kennedy LC, Bickford LR, Lewinski NA, Coughlin AJ, Hu Y, et al. (2011) A new era for cancer treatment: gold-nanoparticle-mediated thermal therapies. Small 7: 169-183.

3. Hughes GA1 (2005) Nanostructure-mediated drug delivery. Nanomedicine 1 : 22-30.

4. Mahmoud MA, El-Sayed MA (2010) Gold nanoframes: very high surface plasmon fields and excellent near-infrared sensors. J Am Chem Soc 132: 12704-12710.

5. Lu W, Huang Q, Ku G, Wen X, Zhou M, et al. (2010) Photoacoustic imaging of living mouse brain vasculature using hollow gold nanospheres. Biomaterials 3: $2617-2626$.

6. Acevedo R, Lombardini R, Halas NJ, Johnson BR (2009) Plasmonic enhancement of Raman optical activity in molecules near metal nanoshells. J Phys Chem A 113: 13173-13183.

7. Blasiak B, van Veggel FCJM, Tomanek B (2013) Applications of Nanoparticles for MRI Cancer Diagnosis and Therapy. Journal of Nanomaterials.

8. Fan X, Zheng W, Singh DJ (2014) Light scattering and surface plasmons on small spherical particles. Light Science and Applications.

9. El-Sayed IH, Huang X, El-Sayed MA (2005) Surface Plasmon Resonance Scattering and Absorption of anti-EGFR Antibody Conjugated Gold Nanoparticles in Cancer Diagnostics:? Applications in Oral Cancer. Nano Letters 5: 829-834.

10. Sun Y, Xia Y (2002) Increased Sensitivity of Surface Plasmon Resonance of Gold Nanoshells Compared to that of Gold Solid Colloids in Response to Environmental Changes. Analytical Chemistry 74: 5297-5305.

11. Liu Y, Shipton MK, Ryan J, Kaufman ED, Franzen S (2007) Synthesis, Stability and Cellular Internalization of Gold Nanoparticles Containing Mixed-PeptidePoly(ethylene glycol) Monolayers. Analytical Chemistry 79: 2221-2229.

12. Han G, Martin CT, Rotello VM (2006) Stability of Gold Nanoparticle-Bound DNA toward Biological, Physical, and Chemical Agents. Chemical Biology and Drug Design 67: 78-82.

13. Albanese A, Tang PS, Chan WC (2012) The effect of nanoparticle size, shape and surface chemistry on biological systems. Annu Rev Biomed Eng 14: 1-16.

14. Zhou J, Ralston J, Sedev R, Beattie DA (2009) Functionalized gold nanoparticles: synthesis, structure and colloid stability. J Colloid Interface Sci 331: $251-262$. 
Citation: Miller A, Adams S, Zhang JZ, Wang L (2016) Investigation into Drug Solubilisation Potential of Sulfonated Calix(4)Resorcinarenes. J Nanomed Nanotechnol 7: 371. doi:10.4172/2157-7439.1000371

15. Mei BC, Susumu K, Medintz IL, Mattoussi H (2009) Polyethylene glycol-based bidentate ligands to enhance quantum dot and gold nanoparticle stability in biological media. Nature Protocols 4: 412-423.

16. Albanese A, Chan WC (2011) Effect of gold nanoparticle aggregation on cell uptake and toxicity. ACS Nano 5: 5478-5489.

17. Schwartzberg AM, Oshiro TY, Zhang JZ, Huser T, Talley CE (2006) Improving Nanoprobes Using Surface-Enhanced Raman Scattering from 30-nm Hollow Gold Particles. Analytical Chemistry 78: 4732-4736.

18. Jain PK, Lee KS, El-Sayed IH, El-Sayed MA (2006) Calculated Absorption and Scattering Properties of Gold Nanoparticles of Different Size, Shape, and Composition: Applications in Biological Imaging and Biomedicine. The Journal of Physical Chemistry B 110: 7238-7248.

19. Nikoobakht B, El-Sayed MA (2003) Surface-Enhanced Raman Scattering Studies on Aggregated Gold Nanorods. The Journal of Physical Chemistry A 107: 3372-3378.

20. Yang W, Gooding JJ, He Z, Li Q, Chen G (2007) Fast colorimetric detection of copper ions using L-cysteine functionalized gold nanoparticles. J Nanosci Nanotechnol 7: 712-716.

21. Chai F, Wang C, Wang T, Ma Z, Su Z (2010) L-cysteine functionalized gold nanoparticles for the colorimetric detection of $\mathrm{Hg} 2+$ induced by ultraviolet light. Nanotechnology 21: 025501.

22. Guan J, Jiang L, Li J, Yang W (2008) pH-Dependent Aggregation of HistidineFunctionalized Au Nanoparticles Induced by Fe3+ Ions. The Journal of Physical Chemistry C 112: 3267-3271.

23. Liang HP, Wan LJ, Bai CL, Liang Jiang L (2005) Gold Hollow Nanospheres:? Tunable Surface Plasmon Resonance Controlled by Interior-Cavity Sizes. The Journal of Physical Chemistry B 109: 7795-7800.

24. Schwartzberg AM, Olson TY, Talley CE, Zhang JZ (2006) Synthesis, Characterization, and Tunable Optical Properties of Hollow Gold Nanospheres. The Journal of Physical Chemistry B 110: 19935-19944.

25. Olson TY, Schwartzberg AM, Orme CA, Talley CE, O'Connell B (2008) Hollow Gold-Silver Double-Shell Nanospheres: Structure, Optical Absorption, and Surface-Enhanced Raman Scattering. J Phys Chem C 112: 6319-6329.

26. Zhang JZ (2010) Biomedical Applications of Shape-Controlled Plasmonic Nanostructures: A Case Study of Hollow Gold Nanospheres for Photothermal Ablation Therapy of Cancer. Journal Physical Chemistry Letters 1: 686-695.

27. Preciado-Flores S, Wang D, Wheeler DA, Newhouse R, Hensel JK, et al. (2010) Highly reproducible synthesis of hollow gold nanospheres with near infrared surface plasmon absorption using PVP as stabilizing agent. Journal of Materials Chemistry 21: 2344-2350.

28. Adams S, Thai D, Mascona X, Schwartzberg AM, Zhang JZ (2014) Key factors Affecting the reproducibility of synthesis and growth mechanism of Near-Infrared absorbing hollow gold nanospheres. Chemistry of Materials 26: 2805-2810.

29. Adams S, Zhang JZ (2016) Unique optical properties and applications of hollow gold nanospheres (HGNs). Coordination Chemistry Reviews.
30. Lopez-Luke T, Wheeler DA, de la Rosa E, Torres-Castro A, Adams SA, et al. (2012) Synthesis, characterization and surface enhanced Raman scattering of hollow gold-silica double shell nanostructures. Biomedical Spectroscopy and Imaging 1: 275-291.

31. Chon H, Lim C, Ha SM, Ahn Y, Lee EK, et al. (2010) On-Chip Immunoassay Using Surface-Enhanced Raman Scattering of Hollow Gold Nanospheres. Analytical Chemistry 82: 5290-5295.

32. Lee S, Chon H, Lee M, Choo J, Shin SY, et al. (2009) Surface-enhanced Raman scattering imaging of HER2 cancer markers overexpressed in single MCF7 cells using antibody conjugated hollow gold nanospheres. Biosensors and Bioelectronics 24: 2260-2263.

33. Melancon MP, Lu W, Yang Z, Zhang R, Cheng Z, et al. (2008) In vitro and in vivo targeting of hollow gold nanoshells directed at epidermal growth factor receptor for photothermal ablation therapy. Molecular Cancer Therapeutics 7: 1730-1739.

34. Kumar R, Maitra AN, Patanjali PK, Sharma P (2005) Hollow gold nanoparticles encapsulating horseradish peroxidase. Biomaterials 26: 6743-6753.

35. Wu GH, Milkhailovsky A, Khant HA, Fu C, Chiu W, et al. (2008) Remotely triggered liposome release by near-infrared light absorption via hollow gold nanoshells. Journal of the American Chemical Society 130: 8175-8177.

36. You J, Zhang R, Zhang G, Zhong M, Liu Y, et al. (2012) Photothermalchemotherapy with doxorubicin-loaded hollow gold nanospheres: A platform for near-infrared light-triggered drug release. Journal Controlled Release 158 319-328.

37. Hahn MA, Singh AK, Sharma P, Brown SC, Moudgil BM (2011) Nanoparticles as contrast agents for in-vivo bioimaging: current status and future perspectives. Analytical and Bioanalytical Chemistry 399: 3-27

38. Turkevich J, Stevenson PC, Hillier J (1951) A study of the nucleation and growth processes in the synthesis of colloidal gold. Discuss Faraday Soc 11: 55-75.

39. Frens $G$ (1973) Controlled nucleation for the regulation of the particle size in monodisperse gold suspensions. National Physics Society 241: 20-22.

40. Chen S, Fang YM, Xiao Q, Li J, Li SB, et al. (2012) Rapid visual detection of aluminium ion using citrate capped gold nanoparticles. Analyst 137: 2021-2023.

41. Tro NJ (2014) Chemistry: A Molecular Approach. (3rdedn) Pearson: Upper Saddle River, NJ.

42. Garrett RH, Grisham CM (2013) Biochemtry. (5thedn) Brooks/Cole, Cengage Learning: Belmont, CA

43. Mocanua A, Cernicab I, Tomoaiac G, Bobosa L, Horovitz O, et al. (2009) Selfassembly characteristics of gold nanoparticles in the presence of cysteine. Colloids and Surfaces A: Physicochemical and Engineering Aspects 338: 93101

44. Burns C, Spendel WU, Puckett S, Pacey GE (2006) Solution ionic strength effect on gold nanoparticle solution color transition. Talanta 60: 873-876.

45. Furia TE (1972) CRC Handbook of Food Additives. (2ndedn) CRC Press. 